\title{
Management of severe low back pain with a focused vibro-percussion wave treatment: A Case Report
}

\author{
Norah Alsalamah ${ }^{1}$ and Lee Bartel ${ }^{2}$ \\ ${ }^{1}$ KKT Orthopedic Spine Center \\ ${ }^{2}$ University of Toronto
}

November 3, 2021

\begin{abstract}
A 49 year-old male with severe low back pain showed multilevel disc bulges with spinal stenosis. After 18 treatments of lowfrequency vibro-percussion stimulation initial VAS pain score of 9.5 reduced to 2.5 and the Rolland-Morris score of 13 reduced to 3. The MRI showed some resolution of L3-L4 and L4-L5.
\end{abstract}

Title: Management of severe low back pain with a focused vibro-percussion wave treatment: A Case Report

Abstract: A 49 year-old male with severe low back pain showed multilevel disc bulges with spinal stenosis. After 18 treatments of low-frequency vibro-percussion stimulation initial VAS pain score of 9.5 reduced to 2.5 and the Rolland-Morris score of 13 reduced to 3. The MRI showed some resolution of L3-L4 and L4-L5.

Key Clinical Message : Focused vibro-percussive sound wave stimulation to the spine known as Khan Kinetic Treatment (KKT) has in this case, and previously through RCT studies, demonstrated improvement in pain and function with no adverse events. KKT is currently available in 13 countries.

Keywords: Case report, Khan Kinetic Treatment, KKT, Low back pain, Spinal stenosis, Vibroacoustic, vibration therapy, sound wave therapy, low frequency sound.

\section{Introduction}

Back pain (BP) is a leading cause of years lived with disability [1], with an estimated 70-85 percent of the population to experience BP at some point in their lives [2]. In North America, disability from BP increased by 14 times the rate of population growth between the years 1950 and 1980 [2]. When measuring overall disease burden, these trends have also occurred on a global scale in all continents, but significantly so in areas such as South Asia, East Asia, North Africa, and the Middle East. Poor education about workplace ergonomic and a lack of effective medical management is thought to be among the main causes of the increase in overall burden [1].

The biggest challenge in treating back pain is determining the actual development of the injury. Spine stability is based on three components: (1) vertebrae and facet joint, ligament, and intervertebral disks; (2) muscles and tendons surrounding the spine; (3) neural systems that control coordinate the active subsystem [3]. A disruption in any one of these three components leads to instability of the spine and injury and pain [3]. Current evidence based treatment modalities focus on a combination of pain medications, exercise, and psychological interventions. Although a minority of patients with chronic BP may qualify for surgery, serious side effects and high costs are an impediment. 
A factor in low back pain may be obesity resulting in stress on the musculoskeletal system. As a result bariatric surgery is conducted in some cases with positive results [4]. However, there is also a known effect on some patients that a gastrectomy is followed by increasing low back pain resulting from the change in intra-abdominal pressure, spinal loading, and a decrease in stability of the spine [5].

Alternative treatment modes are, consequently, being explored. One novel treatment being used successfully for many patients is the KKT Treatment (Khan Kinetic Treatment). This non-invasive approach provides orthopaedic spinal treatment with focused sound waves. The KKT device delivers digitally derived personalized low frequency sound waves within the audible range $(50-100 \mathrm{~Hz})$ focused through a stylus onto particular locations on the spine. This sound wave therapy is a safe therapy that has demonstrated the ability to decrease pain, reduce anxiety, reduce the symptoms of illness, and generally promote health in many patients [6]. Research has shown that the musculoskeletal effects of the novel sound wave therapy result in pain reduction and spinal alignment but also include increased mRNA expression of key proteins for spinal health and a cellular environment conducive to ligament repair [6-9].

Low back pain is a major problem that increases patients' suffering and increases health care costs. Treatment for low back pain must control pain perception, improve alignment of the spine, and stimulate the healing of ligamentous structures. Research into the sound wave therapy has theorized that the KKT treatment addresses these crucial requirements of effective back treatment by: (1) stimulating intervertebral discs biosynthesis; (2) Correcting abnormal intervertebral joint rotation (mean axis of rotation, MAR); (3) Gating pain transmission by activating key circuitry in the spinal cord; (4) Minimizing asymmetrical loads on the spine by relaxing paraspinal muscles; and (5) increasing coordination of muscle groups that play a critical role in stabilization of the spine [8,9]. It is very important to improve our management approach of low back pain to help reduce the risk and costs of such pain. The following case report of an adult patient who presented with low back pain illustrates the effect of the KKT treatment.

\section{Case presentation}

The patient was a 49-year-old Saudi man who presented to the KKT Clinic at Qassim with severe low back pain radiating to both lower limbs. He walked into the clinic with an antalgic gait, an abnormal pattern of walking secondary to pain that ultimately causes a limp, and was holding walls while changing direction. His history showed that he developed back pain in 2016 and his back pain became more severe in 2018 when he weighed $130 \mathrm{Kg}$. It affected his work to the point that he was off work for a year. In an attempt to relieve the back pain he underwent a gastrectomy in February 2020. Before the surgery his VAS was 6 or $7 / 10$ and after the surgery it became $9 / 10$. He lost $35 \mathrm{~kg}$ but the increase in back pain following the surgery caused him to stay in bed for a month. Post surgery he also developed a deficit in vitamin B12, folic acid, and iron. He took supplements and his vitamin and mineral values returned to normal. When he presented at the clinic he had low back pain radiating to both lower limbs, pain aggravated by standing, sitting, and walking, and he experienced stress, a sleep disorder, and frequent headaches, but had no history of other health disorders or accidents. Prior to presenting at the clinic he had used pain medications and standard treatments including physical therapy, osteopathy, and massage but none of these improved his pain or sleep. The patient has given informed written consent for his images and other clinical information to be reported in this journal.

\section{Physical examination and diagnosis}

At the initial examination the patient had a Visual Analogue Scale (VAS) for pain score of 9/10 and RolandMorris (RM) score of 13 points. Magnetic resonance imaging (MRI) of the lumbar spine showed the presence of multilevel disc bulges with mild spinal canal stenosis (Figure1). Other diagnoses considered were multilevel lumbar disc disorder with radiculopathy in the lumbosacral region.

A physical examination was performed to determine the severity of symptoms and possible causes. Palpation of the patient revealed severe tenderness in the mid-thoracic and lumbosacral spine area, moderate to severe limitation of trunk flexion due to pain, and positive straight leg raise (SLR) test on both sides. 
As part of the diagnosis and before each treatment a series of physical and neurological tests were conducted: (1) range of motion (ROM) of the cervical spine; (2) degree of tilt (to a quarter degree with a set of callipers) of the shoulder and pelvis in the coronal plane; (3) discrepancy of supine leg length; (4) coordinated response to resistance of arm and leg; and (5) tender lesions assessment with deep spinal palpation. If results of the assessment before the treatment showed departure from normal, the indicated assessments were repeated after each treatment application to determine response. At the initial examination the patient presented with: (1) normal cervical range of motion (ROM) to both sides; (2) the shoulder tilt deviation to the right by one degree and the pelvic tilt deviated to the right by 0.5 degrees; (3) arm coordination response to resistance was (5/5) bilaterally; (4) leg coordination was reduced on the left (3/5) and the right (4/5); (5) supine leg length was $1 \mathrm{~cm}$ shorter on the right side compared to the left side; and (6) and painful tender lesions were found at 6 points along the spine.

\section{Treatment plan and follow-up}

The KKT Treatment device is a sound transducer-driven, vibratory stylus, impulse delivery mechanism. The device head is mounted on a flexibly positionable armature on a fixed stand [3]. The head of the device, moveable in three dimensions, is positioned by the clinician and then fixed in location at the prescribed spinal location and angle. The stylus is pressure sensitive and so protects patient safety by being collapsible if the patient moves out position (Figure 2) [3]. The device's sound transducer generates waveforms at patient assessment-determined frequencies that are transmitted through to the spine causing vibration of the vertebrae and minor stretching of the soft tissues. At the initial assessment, treatment parameters are determined from digital data captured through X-rays of the spine. Table 1 indicates the anatomical locations at which the treatment was applied in this case and the number of pulses used during each session. The treatment plan for the patient in this report was for 18 sessions of KKT ( 3 times a week for the first 3 weeks and then about once per week). After every six sessions, the patient completed the VAS for pain. The Rolland-Morris test was completed at the first session and after the last session.

\section{Outcomes}

During the course of the KKT treatments the patient reported gradual reduction of pain. The 6 painful tender lesions at the initial assessment were reduced to 5 by session 9 , to 4 by session 13 , and to 0 by session 18. The VAS pain score was 9.5 at the initial assessment and was reduced to 2.5 by the final treatment session. The initial Rolland-Morris score of 13 was reduced to 3 points by the final session. The patient experienced improvement in his daily activity of life and sleep as well as at work due to his decreased pain. In addition, the patient showed correction in clinician-observed biomechanics of his spine and body. Shoulder and pelvic tilt steadily improved with KKT treatment becoming and remaining neutral from the 10th treatment. Cervical ROM reached full normal function by the 13th treatment as well as upper limb coordination. After the completed treatment sessions, the patient's MRI showed some resolution of L3-L4 and L4-L5 (Figure 3). No adverse events related to the treatment were experienced by the patient.

\section{Discussion}

The case described in this paper demonstrates the KKT treatment focused on low back pain. Research literature reveals that even mild LBP results in significant functional loss and decreased quality of life. LBP is the second most common cause for consulting a doctor. Typical treatment of LBP includes various psychological, medical, and physical therapies. When these treatment options fail, the patient often chooses the last option - spinal surgery. However, spinal surgery may not produce symptomatic relief in the long-term and so the patient returns to daily activity with complications. The KKT approach, treating the patient with sound wave stimulation $[3,6,7,9]$, presents a non-invasive alternative. This case study of a middle-aged adult patient who presented with low back pain radiating to both lower limbs treated with KKT is consistent with evidence previously published $[3,7,9,10]$. After the patient received 18 sessions over a period of 13 weeks, he reported improvement in disability and pain scores, sleep, mood, quality of life, and work performance, No adverse effects were observed. Improvement in the lumbar spine was noted on MRI imaging.

\section{Conclusion}


In conclusion, the KKT treatment for lumbar disc disorder is shown useful. The patient experienced symptomatic relief and, as a result, improved quality of life, mood, and work performance. Some of the positive structural changes were also seen on MRI. The level of improvement the patient experienced in his condition and the renewed level of confidence enabled him to return to activity without any symptoms.

\section{Acknowledgements}

Dr. Hussain Al Dera for facilitating the selection of the case. Dr. Aslam Khan for advice in selecting the case and comments on the draft of the manuscript. Ahlam Aldawas and Ashwag Alnwamsy for assistance in conducting the treatment of the patient.

\section{References}

1. Driscoll T, Jacklyn G, Orchard J, et al. The global burden of occupationally related low back pain: estimates from the Global Burden of Disease 2010 study. Ann Rheum Dis. 2014 Jun; 73(6): 975-81. doi: 10.1136/annrheumdis-2013-204631. Epub 2014 Mar 24. PMID: 24665117.

2. Andersson GB. Epidemiological features of chronic low-back pain.Lancet 354; 1999: 581-585.

3. Desmoulin G, Yasin N and Chen D. Spinal mechanisms of pain control. Clin J Pain, 2007; 23(7): 576-585.

4. Koremans FW, Chen X, Das A, et al. Changes in back pain scores after bariatric surgery in obese patients: A systematic review and meta-analysis. J Clin Med . 2021;10(7):1443. Published 2021 Apr 1. doi:10.3390/jcm10071443

5. Skaf G, Elias E, Nasser Z, et al. Worsening back pain after bariatric surgery: The impact of acute weight loss on the spine. Remed Open Access. 2017; 2: 1046.

6. Bartel L and Mosabbir A. Possible mechanisms for the effects of sound vibration on human health. Healthcare 2021; 9(5): 597.

7. Desmoulin G, Hewitt C and Hunter C. Disc strain and resulting positive mRNA expression from application of a noninvasive treatment. Spine 2011; 36(14): E921-E928.

8. Desmoulin G, Reno C and Hunter C. Free axial vibrations at 0 to $200 \mathrm{~Hz}$ positively affect extracellular matrix messenger ribonucleic acid expression in bovine nucleus pulposi. Spine 2010; 35(15): 1437-1444.

9. Desmoulin G, Szostek J, Khan A, et al. Spinal intervention efficacy on correcting cervical vertebral axes of rotation and the resulting improvements in pain, disability and psychosocial measures. . J Musculoskelet Pain 2011; 20(1), pp.31-40.

10. Desmoulin GT, Yasin NI and Chen DW. Initial results using Khan Kinetic Treatment as a low back pain treatment option. J Musculoskelet Pain 2007; 15(3): 91-102.

\section{Tables}

Table 1. Location of treatment and Number of pulses .

\section{Treatment location and pulses}

\begin{tabular}{lllll}
\hline Tx\# & Cervical & Lumbar & Sacrum/iliac & Abdomen \\
1 & Right C1 80 & & & \\
2 & Right C1 80 & & & \\
3 & Right C1 80 & & & \\
4 & Right C1 80 & & & \\
5 & Right C1 80 & & & \\
6 & Right C1 80 & & & \\
7 & Right C1 80 & L5 60 & Rt PSIS 80 Lt PSIS 80 & \\
8 & Right C1 80 & L5*60 & Rt PSIS 80 Rt Trochanter 80 &
\end{tabular}




\begin{tabular}{|c|c|c|c|c|}
\hline 9 & Right C1 80 & $\mathrm{~L} 5 * 60$ & Rt PSIS 80 & \\
\hline 10 & Right C1 80 & $\mathrm{~L} 5 * 60$ & Rt PSIS 80 Lt PSIS 80 & \\
\hline 11 & Right C1 80 & $\mathrm{~L} 5 * 60$ & Rt PSIS 80 Lt PSIS 80 & LLQ 40 \\
\hline 12 & Right C1 80 & $\mathrm{~L} 5 * 60$ & Rt PSIS 80 & LLQ 40 \\
\hline 13 & Right C1 80 & $\mathrm{~L} 5^{*} 60$ & Rt PSIS 80 & \\
\hline 14 & Right C1 80 & $\mathrm{~L} 5^{*} 60$ & Rt PSIS 80 & \\
\hline 15 & Right C1 80 C5*40 & $\mathrm{L} 5^{*} 60$ & & \\
\hline 16 & Right C1 80 & $\mathrm{~L} 5^{*} 60$ & & \\
\hline 17 & Right C1 80 & $\mathrm{~L} 5 * 60$ & & \\
\hline 18 & Right C1 80 & $\mathrm{~L} 5 * 60$ & & \\
\hline
\end{tabular}

Lft $=$ left; PSIS $=$ Posterior superior iliac spine; $\mathrm{Rt}=$ right; $\mathrm{Tx}=$ Treatment, $\mathrm{LLQ}=$ Lateral Lower Quadrant "Right" indicates application of the stimulus from the right side, e.g., Right C1 indicates the stimulus pulses were applied on the right side of $\mathrm{C} 1$. L or left indicates application on the left side. Asterix * indicates application at the midline.

\section{Figures}




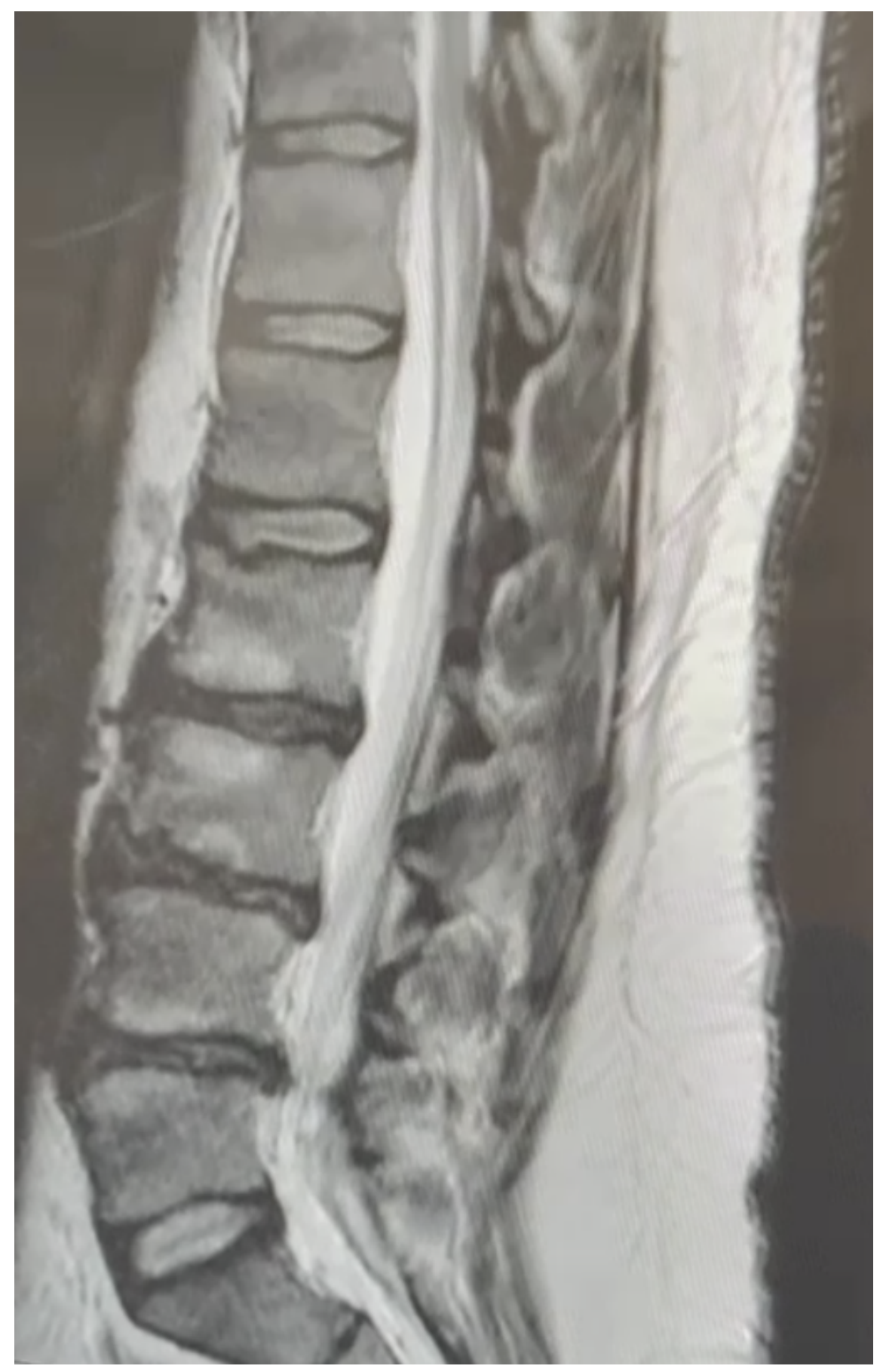

Figure 1. MRI of the lumbar spine before treatment showing bulging of the discs between L5- L2 region. 


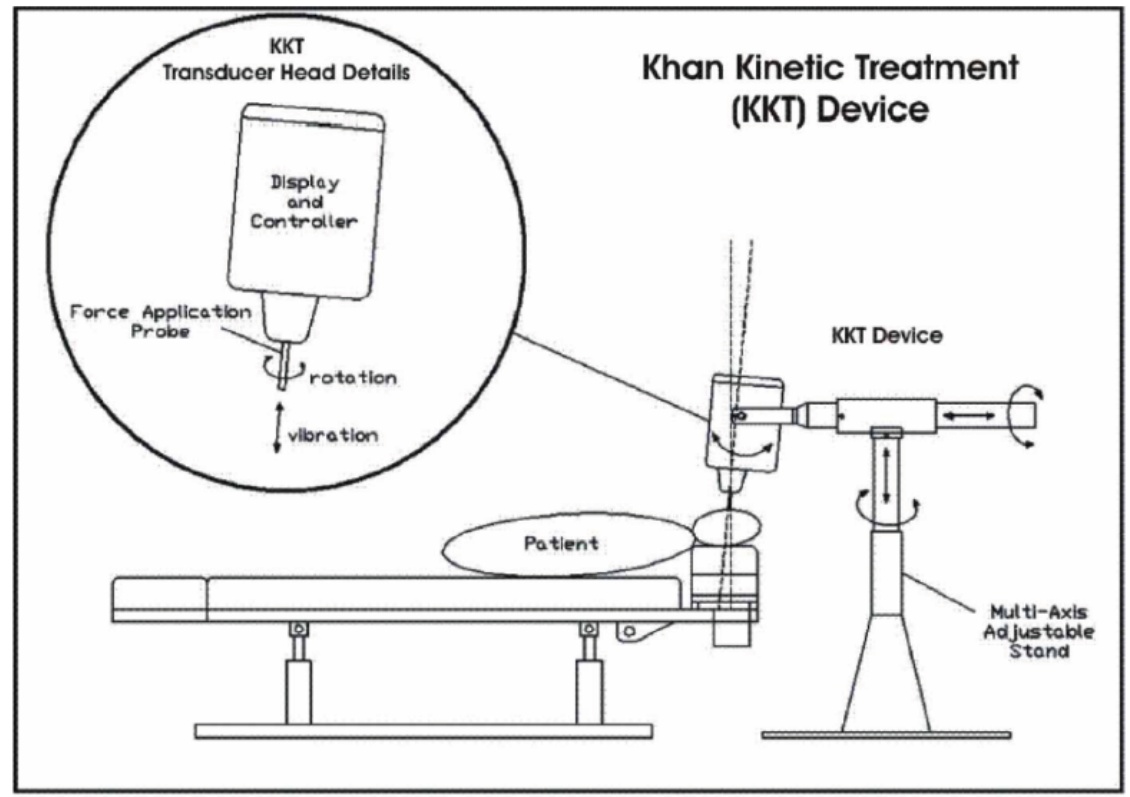

Figure 2. KKT device. 


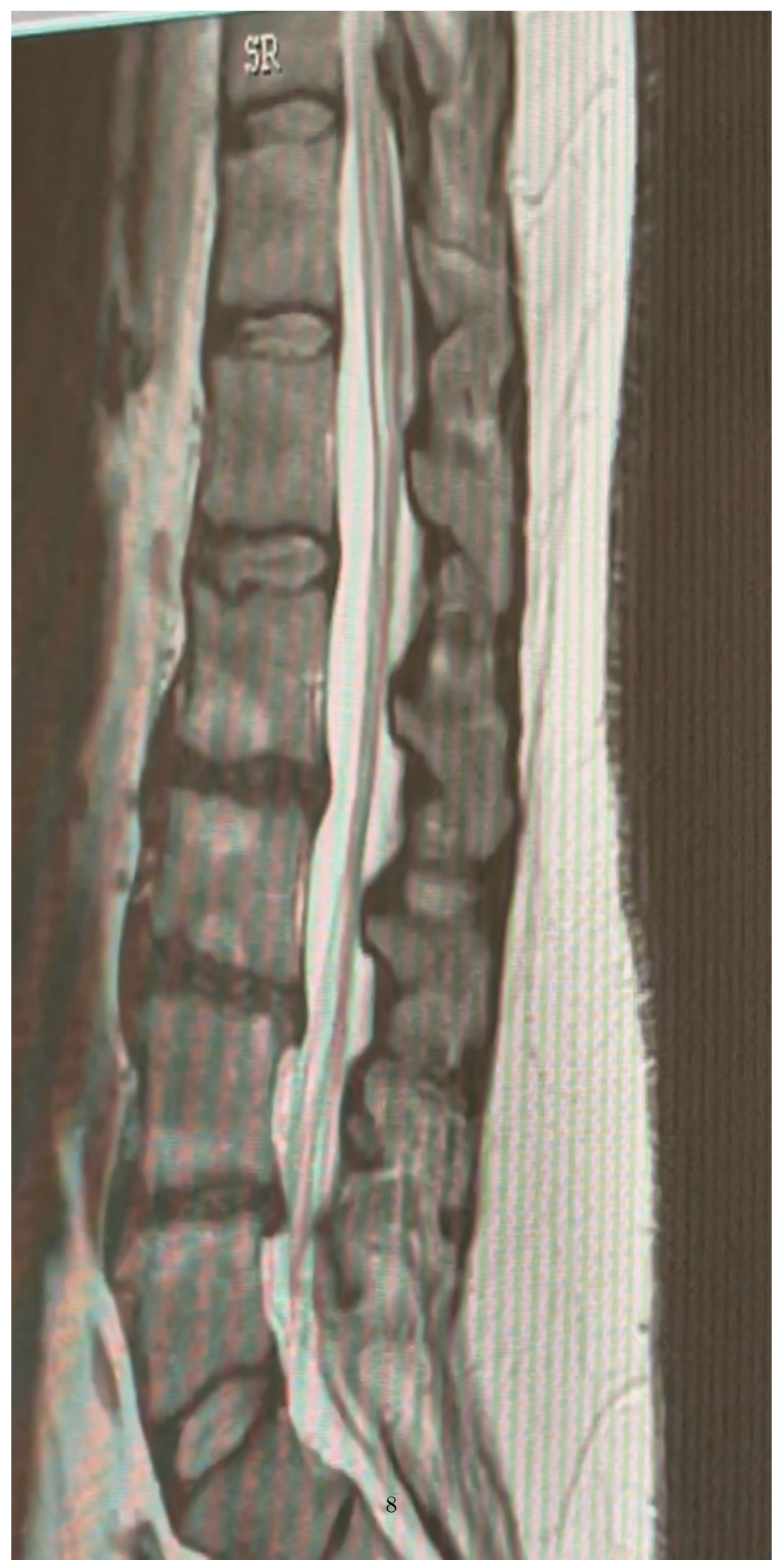


Figure 3. MRI of the lumbar spine after treatment showing reduced bulging of discs between L5-L2 